Supporting Information

\title{
MICROMOTORS AS “MOTHERSHIPS": A CONCEPT FOR THE TRANSPORT, DELIVERY, AND ENZYMATIC RELEASE OF MOLECULAR CARGO VIA NANOPARTICLES
}

Tomáš Kroupa, ${ }^{\dagger}$ Soňa Hermanová, ${ }^{\dagger}, \ddagger$ Carmen C. Mayorga-Martinez, ${ }^{\ddagger}$ Filip Novotný, ${ }^{\ddagger}$ Zdeněk Sofer, ${ }^{\ddagger}$ and Martin Pumera ${ }^{\ddagger} \$^{*}$

Department of Polymers, Faculty of Chemical Technology, University of Chemistry and Technology Prague, Technická 5, 16628 Prague, Czech Republic.

‡ Center for Advanced Functional Nanorobots, Department of Inorganic Chemistry, Faculty of Chemical Technology, University of Chemistry and Technology Prague, Technická 5, 16628 Prague, Czech Republic. \# Department of Chemical and Biomolecular Engineering, Yonsei University, 50 Yonsei-ro, Seodaemun-gu, Seoul 03722, Korea

\$ Future Energy and Innovation Laboratory, Central European Institute of Technology, Brno University of Technology, Purkyňova 656/123, Brno, CZ-616 00, Czech Republic

*E-mail: martin.pumera@vscht.cz 
Table S1 Polymerization results and OH-PCL-SH characterization

\begin{tabular}{ccccc}
\hline $\begin{array}{c}\text { monomer:CALB } \\
30: 1\end{array}$ & $\begin{array}{c}\text { CALB } \\
{[\mathrm{mg}]}\end{array}$ & $\begin{array}{c}\mathrm{M}_{\mathrm{n}}(\mathrm{SEC})^{\mathrm{a}} \\
{\left[\mathrm{g} \cdot \mathrm{mol}^{-1}\right]}\end{array}$ & $\mathrm{Ð}(\mathrm{SEC})$ & $\begin{array}{c}\text { Crystallinity }^{\mathrm{b}} \\
{[\%]}\end{array}$ \\
\hline 50 & 10000 & 1.5 & 58 \\
\hline
\end{tabular}

a SEC analysis: SEC chromatograph (Waters Breeze) with two columns (PSS Lux, PL Gel Mixed C (mixed bed, $5 \mu \mathrm{m}$ ) and refractometric detector was applied to determine molar mass distribution. Analysis was done in THF as mobile phase with the rate $1 \mathrm{~mL} \cdot \mathrm{min}^{-1}$ at $35^{\circ} \mathrm{C}$.

${ }^{b}$ DSC analysis was performed on DSC Q100 instrument (TA Instrument). Crystallinity was calculated according to $2^{\text {nd }}$ heating run, using $139.5 \mathrm{~J} \cdot \mathrm{g}^{-1}$ as a reference for $100 \%$ crystalline PCL. ${ }^{1}$

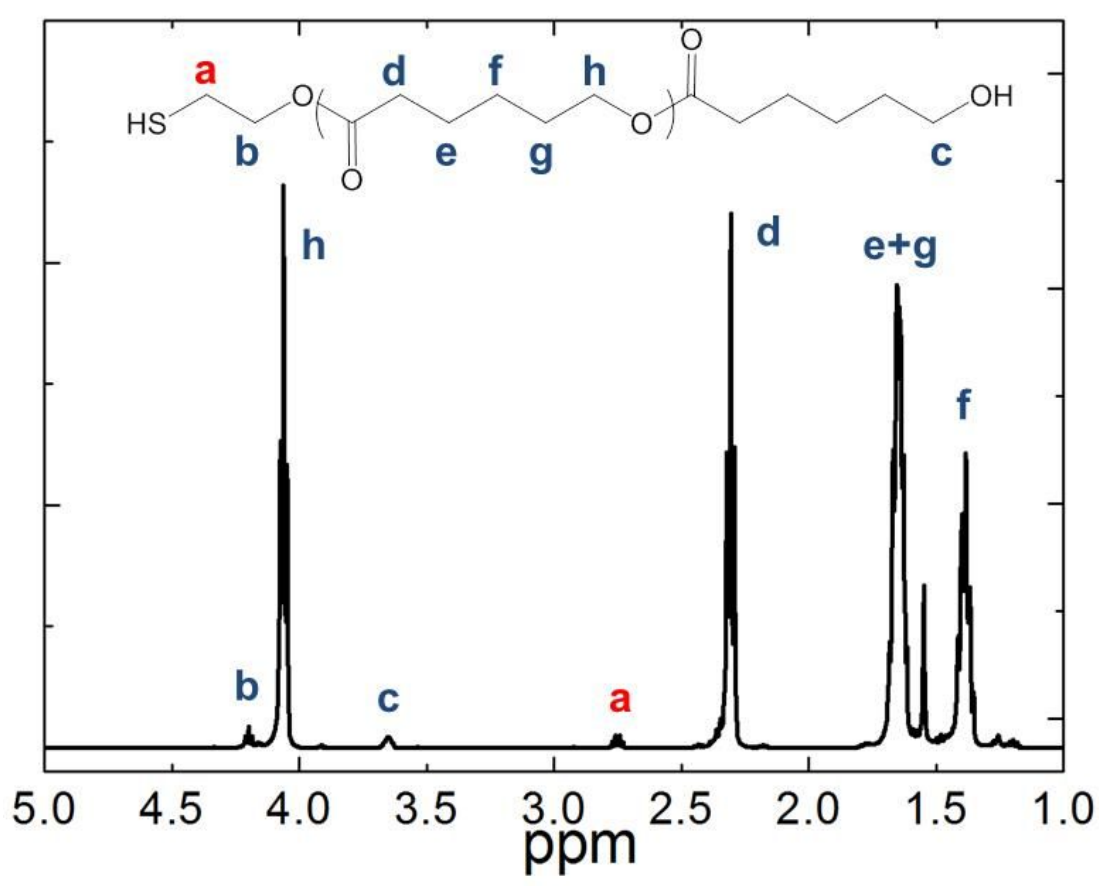

Figure $\mathrm{S1}{ }^{1} \mathrm{H}$ NMR spectrum of $\mathrm{OH}-\mathrm{PCL}-\mathrm{SH}$ in $\mathrm{CDCl}_{3}, 25^{\circ} \mathrm{C}: 1.38 \mathrm{ppm}[2 \mathrm{H}$, $\left.\mathrm{CO}\left(\mathrm{CH}_{2}\right)_{2} \mathrm{CH}_{2}\left(\mathrm{CH}_{2}\right)_{2} \mathrm{O}-\right] ; 1.65$ ppm (4H, $\left.-\mathrm{COCH}_{2} \mathrm{CH}_{2} \mathrm{CH}_{2} \mathrm{CH}_{2} \mathrm{CH}_{2} \mathrm{O}-\right) ; 2.31$ ppm [2H, $\left.\mathrm{COCH}_{2^{-}}\left(\mathrm{CH}_{2}\right)_{4} \mathrm{O}-\right] ; 2.76 \mathrm{ppm}\left(2 \mathrm{H}\right.$, HS- $\left.\mathrm{CH}_{2} \mathrm{CH}_{2-}\right) ; 3.65 \mathrm{ppm}\left[2 \mathrm{H},-\mathrm{CO}\left(\mathrm{CH}_{2}\right)_{4} \mathrm{CH}_{2} \mathrm{OH}\right]$; 4.06 ppm $\left[2 \mathrm{H},-\mathrm{CO}\left(\mathrm{CH}_{2}\right)_{4}-\mathrm{CH}_{2} \mathrm{O}-\right] ; 4.20$ ppm $\left(2 \mathrm{H}, \mathrm{HS}-\mathrm{CH}_{2}-\mathrm{CH}_{2} \mathrm{O}-\right)$ 


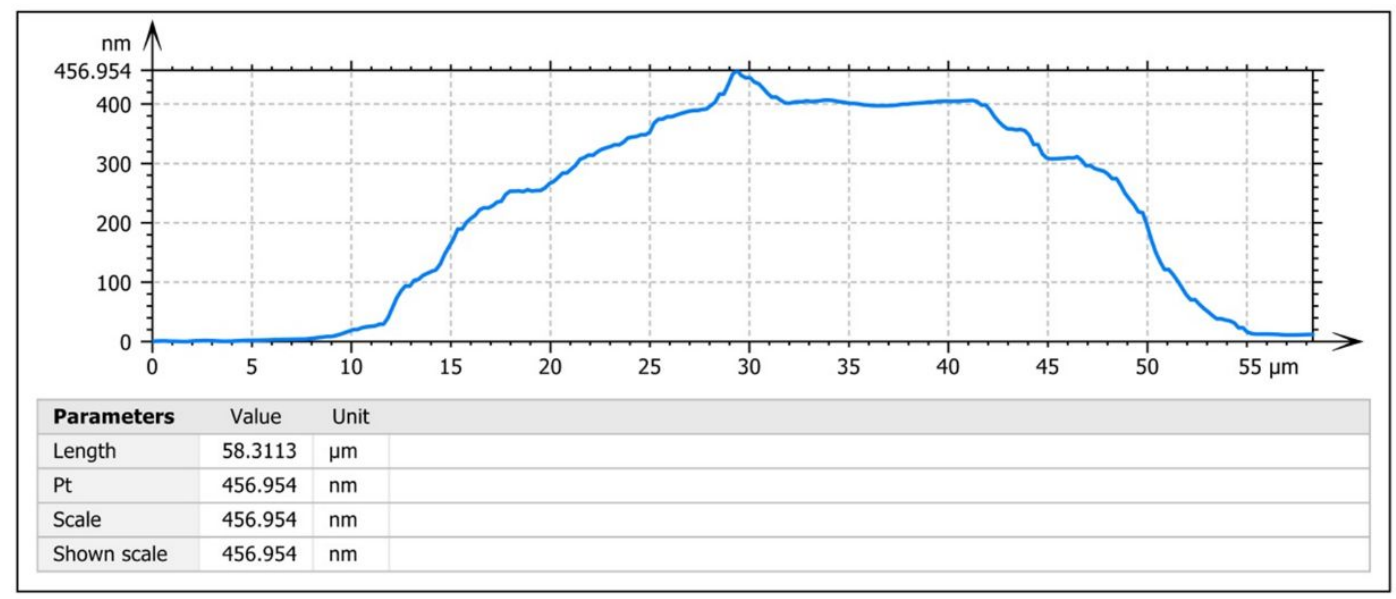

Figure S2. Height profile of Pt/PCL-SH micromotors by optical profilometer (Senofar S neox).

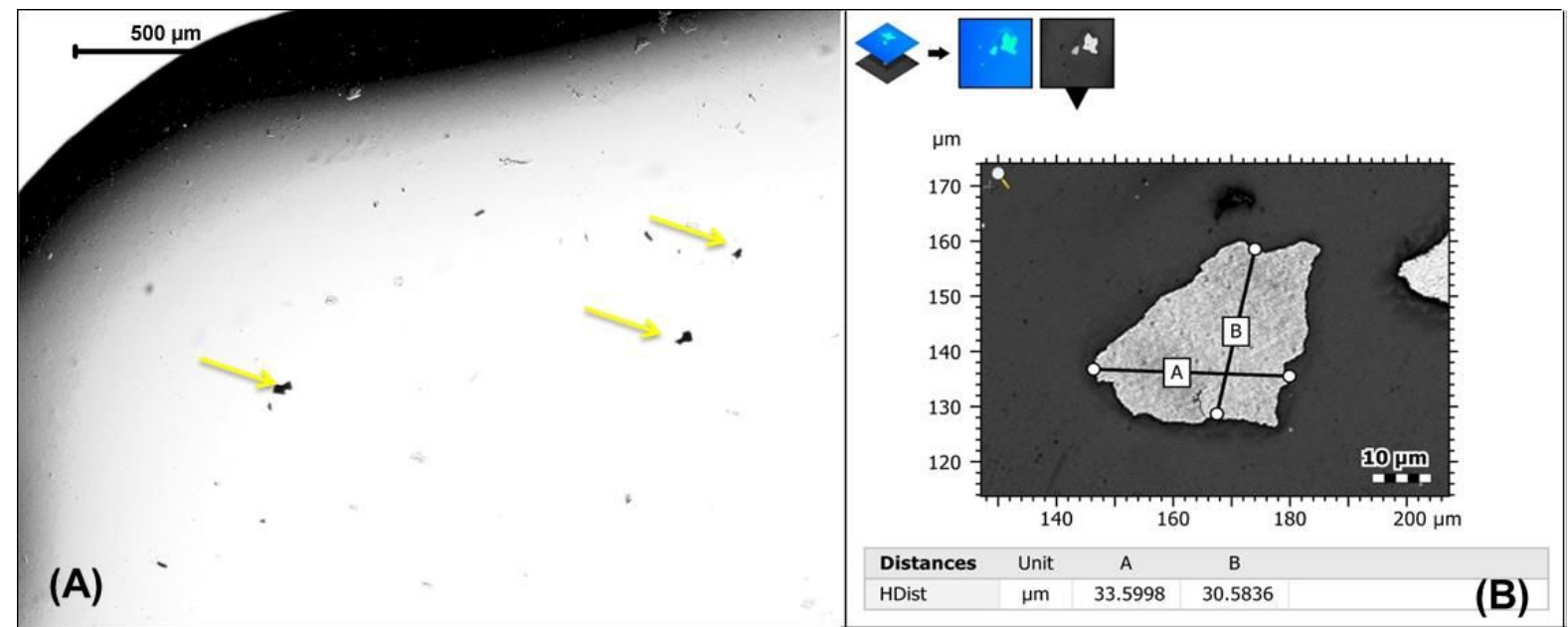

Figure S3. Images of Pt/PCL-SH micromotors. A) Sheet-like micromotors in a water drop (optical microscopy). B) Representative sheet-like micromotor (optical profilometer, Senofar S neox). 

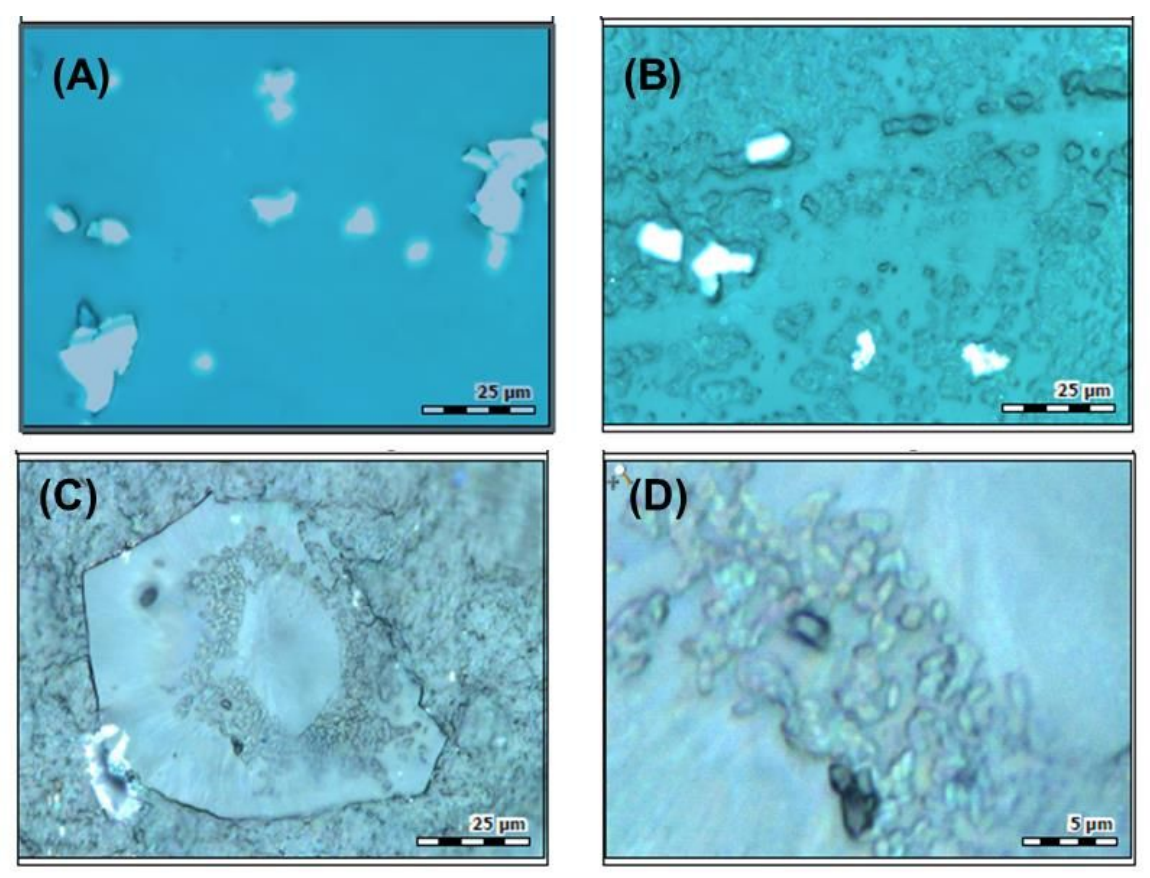

Figure S4. Optical profilometer image of PCL microsheets and micromotors. A) Pt/PCL micromotors before the incubation. B) Pt/PCL micromotors after incubating with lipase for 6 days (stock solution $10 \mathrm{mg} / \mathrm{mL}$. C) PCL sheets free of Pt after the incubation with enzyme. D) Detail of Pt-free PCL pieces resulted from hydrolytic scission.

\section{References}

1. Crescenzi, V.; Manzini, G.; Calzolari, G.; Borri, C., Thermodynamics of fusion of poly- $\beta$-propiolactone and poly- $\epsilon$-caprolactone. comparative analysis of the melting of aliphatic polylactone and polyester chains. European Polymer Journal 1972, 8 (3), 449-463. 